\title{
A close-up view of a bipolar jet: Sub-arcsecond near-infrared imaging of the high-mass protostar IRAS 20126+4104^
}

\author{
R. Cesaroni ${ }^{1}$, F. Massi ${ }^{1}$, C. Arcidiacono ${ }^{1,2}$, M. T. Beltrán ${ }^{1}$, D. McCarthy ${ }^{3}$, C. Kulesa ${ }^{3}$, K. Boutsia ${ }^{4}$, D. Paris ${ }^{4}$, \\ F. Quirós-Pacheco ${ }^{1}$, and M. Xompero ${ }^{1}$
}

1 INAF, Osservatorio Astrofisico di Arcetri, Largo E. Fermi 5, 50125 Firenze, Italy e-mail: [cesa; fmassi; mbeltran; fquiros;marco] @arcetri .astro.it

2 INAF, Osservatorio Astronomico di Bologna, Via Ranzani 1, 40127 Bologna, Italy e-mail: carmelo.arcidiacono@oabo.inaf.it

3 Steward Observatory, The University of Arizona, 933 N. Cherry Ave., Tucson, AZ-85721, USA e-mail: dwmccarthy@gmail.com, ckulesa@as.arizona.edu

4 INAF, Osservatorio Astronomico di Roma, via Frascati 33, 00040 Monteporzio, Italy e-mail: [konstantina.boutsia;diego.paris]@oa-roma.inaf.it

Received 22 October 2012 / Accepted 14 November 2012

\begin{abstract}
Context. The formation of OB-type stars up to (at least) $140 M_{\odot}$ can be explained via disk-mediated accretion and in fact growing observational evidence of disk-jet systems is found in high-mass star-forming regions.

Aims. With the present observations we wish to investigate at sub-arcsecond resolution the jet structure close to the well studied high-mass protostar IRAS 20126+4104, which is known to be surrounded by a Keplerian disk.

Methods. Adaptive optics imaging of the $2.2 \mu \mathrm{m}$ continuum and $\mathrm{H}_{2}$ and $\mathrm{Br} \gamma$ line emission have been performed with the Large Binocular Telescope, attaining an angular resolution of $\sim 90$ mas and an astrometric precision of $\sim 100$ mas.

Results. While our results are consistent with previous $K$-band images by other authors, the improved (by a factor $\sim 3$ ) resolution allows us to identify a number of previously unseen features, such as bow shocks spread all over the jet structure. Also, we confirm the existence of a bipolar nebulosity within $1^{\prime \prime}$ from the protostar, prove that the emission from the brightest, SE lobe is mostly due to the $\mathrm{H}_{2}$ line, and resolve its structure.

Conclusions. Comparison with other tracers such as masers, thermal molecular line emission, and free-free continuum emission proves that the bipolar nebulosity is indeed tracing the root of the bipolar jet powered by the deeply embedded protostar at the center of the Keplerian disk.
\end{abstract}

Key words. stars: formation - ISM: individual objects: IRAS 20126+4104 - ISM: jets and outflows

\section{Introduction}

In the context of star formation, high-mass stars are commonly defined as those exceeding $\sim 8 M_{\odot}$. While the value of such a threshold may depend on a number of physical parameters, its existence is determined by the simple fact that the radiation pressure of luminous stars may halt the accretion process, thus questioning the possibility that high-mass stars can form through (disk) accretion like their low-mass siblings. However, recent observational and theoretical studies indicate that diskjet systems may be a viable solution to explain the formation of OB-type stars. On the one hand, growing evidence of circumstellar (Keplerian) disks with associated bipolar jets along the rotation axis is being obtained by means of high angular resolution

* Based on observations carried out with the Large Binocular Telescope. The LBT is an international collaboration among institutions in the United States, Italy and Germany. LBT Corporation partners are: The University of Arizona on behalf of the Arizona university system; Istituto Nazionale di Astrofisica, Italy; LBT Beteiligungsgesellschaft, Germany, representing the Max-Planck Society, the Astrophysical Institute Potsdam, and Heidelberg University; The Ohio State University, and The Research Corporation, on behalf of The University of Notre Dame, University of Minnesota, and University of Virginia. imaging at (sub)mm and IR wavelengths (see e.g. Cesaroni et al. 2007; Kraus et al. 2010; de Wit et al. 2010, 2011). On the other hand, numerical models confirm that disk-mediated accretion can explain the formation of stars as massive as $\sim 140 M_{\odot}$ (Krumholz et al. 2009; Kuiper et al. 2010, 2011).

The next step of this study will be the analysis of the accretion (through the disk) and ejection (through the associated jet) processes close to the (proto)star. While interferometric observations at (sub)mm wavelengths have been traditionally used to investigate the heavily extincted surroundings of deeply embedded OB (proto)stars, substantial progress has been recently made in the near-IR regime. In particular, adaptive optics provides us with angular resolutions $\lesssim 100$ mas in the near-IR, superior to those attainable until now in the (sub)mm. Although at short wavelengths one does not expect to detect the (proto)star at the center of the disk, if the line of sight is favorable it should be possible to see the scattered stellar photons from the innermost part of the disk/jet and trace the jet itself through the $\mathrm{H}_{2}$ line emission.

With this in mind, we have performed LBT/PISCES AO observations of the $2.2 \mu \mathrm{m}$ continuum and $\mathrm{H}_{2} v=1-0 \mathrm{~S}(1)$ line emission towards the high-mass protostar IRAS $20126+4104$. This is a well studied object of $\sim 10^{4} L_{\odot}$, associated with 
a disk-jet/outflow system and located at a distance of $1.64 \pm$ $0.05 \mathrm{kpc}$ (Moscadelli et al. 2011). The disk of $\sim 1000 \mathrm{AU}$ is undergoing Keplerian rotation consistent with a stellar mass of $\sim 7 M_{\odot}$ (Cesaroni et al. 2005). The jet/outflow has been imaged from the small (a few $100 \mathrm{AU})$ to the large $(\sim 0.5 \mathrm{pc})$ scale using a variety of tracers $\left(\mathrm{H}_{2} \mathrm{O}, \mathrm{H}_{2}\right.$, free-free continuum, $\mathrm{SiO}$, $\mathrm{HCO}^{+}$, CO, etc.; Cesaroni et al. 1997, 1999; Lebrón et al. 2009; Moscadelli et al. 2000, 2005, 2011; Su et al. 2007; Caratti o Garatti et al. 2008; Hofner et al. 1999, 2007) and is found to undergo precession about the rotation axis of the disk (Shepherd et al. 2000; Cesaroni et al. 2005). Near-IR images in the $K, L$, and $M^{\prime}$ bands with 0.3 resolution were obtained by Sridharan et al. (2005; hereafter SWF), unveiling the existence of a bipolar nebulosity along the direction of the jet/outflow. At $K$ band this bipolar structure outlines a dark lane which is interpreted by the authors as the disk silhouette, whereas in the other bands the authors claim to detect emission from the disk as well as from a nearby companion. However, later observations performed by De Buizer (2007) with an 8-m class telescope in the mid-IR have not confirmed the presence of emission from the disk and questioned the presence of the secondary source. In contrast, more recently, de Wit et al. (2009) imaged the source with Subaru at $24.5 \mu \mathrm{m}$, revealing strong, compact emission that could originate from the dark lane.

The scope of our new observations is to significantly improve (by a factor $\sim 3$ ) on the angular resolution of previous IR images and thus shed light on the nature of the structures observed within a few 1000 AU from the IRAS 20126+4104 protostar.

\section{Observations and data reduction}

The data were collected on June 21, 2012, using the PISCES Near Infrared Camera (McCarthy et al. 2001) installed at the focal plane of the First Light Adaptive Optics system (Esposito et al. 2010, 2011) of the Large Binocular Telescope. This allows a field of $21^{\prime \prime} \times 21^{\prime \prime}$ to be imaged with a plate scale of $\sim 0.02 \mathrm{arcsec} / \mathrm{pixel}$. The observations were carried out through the $K_{\mathrm{s}}$ broad-band filter (at $\left.2.12 \mu \mathrm{m}\right)$, and the $\mathrm{H}_{2}(2.12 \mu \mathrm{m})$ and $\operatorname{Br} \gamma(2.17 \mu \mathrm{m})$ narrow-band filters. We used a star with $R \simeq$ $14 \mathrm{mag}\left(\alpha(\mathrm{J} 2000)=20^{\mathrm{h}} 14^{\mathrm{m}} 25^{\mathrm{s}} .069, \delta(\mathrm{J} 2000)=+41^{\circ} 13^{\prime} 32^{\prime \prime} \cdot 23\right)$, $\sim 12^{\prime \prime}$ west of the main source, as the reference for the AO loop, closed using 66 modes.

The on-source frames were first flat-fielded, sky subtracted with a median of the sky frames and corrected for the optical PISCES distortion using the "drizzle" IRAF routine. Finally, they were registered and combined together. The final images were calibrated using the only two stars from the 2MASS catalog falling inside the field. In principle, this makes the calibration prone to stellar variability, but we checked that the measured flux from the jet in the $\mathrm{H}_{2}$ line is consistent within $30 \%$ with the values reported by Cesaroni et al. (1997). To maximize the astrometric precision, we retrieved a much larger $K$ image of the field from NICS at the TNG (better resolved than 2MASS) and used a number of stars within $\sim 2^{\prime}$ of the jet, to obtain a positional fit to the corresponding 2MASS stars. We used the astrometric solution (through IRAF routines) to refine the absolute positions of the pair of 2MASS stars inside the PISCES field, yielding a correction of only a fraction of arcsec to the coordinates given in the 2MASS catalog. The astrometric precision of the final images should be better than $\sim 0{ }^{\prime} 1$ and the point spread function is $\sim 0$.'09.

The $\mathrm{Br} \gamma$ image does not display line emission, so it was used only to estimate the continuum contribution inside the $\mathrm{H}_{2}$ filter and will not be mentioned anymore in the following sections.
A continuum-free $\mathrm{H}_{2}$ image was constructed by subtracting the Br $\gamma$ image after shifting and scaling.

\section{Results and discussion}

\subsection{The large scale structure of the jet}

Our results are illustrated in Figs. 1 and 2. The former is a composite image where the three filters have been combined. The latter shows the continuum-subtracted $\mathrm{H}_{2}$ line and the broad band $\left(K_{\mathrm{S}}\right)$ images separately. Even by-eye comparison between the two panels of this figure clearly reveals that the $K_{\mathrm{s}}$ image contains substantial continuum emission. This outlines a bipolar nebulosity presumably caused by scattered stellar photons along the outflow axis, in agreement with the findings of Cesaroni et al. (1997) - see their Fig. 12. More interesting is the image of the $\mathrm{H}_{2}$ line emission (Fig. 2b). Here one sees that the whole emitting region is spotted with bow shocks, spanning a broad range of sizes. This fact as well as the highly irregular structure of the jet lobes suggests that the ejection phenomenon is episodic, irregular in time, and asymmetric with respect to the star. Worth of notice is also the wiggling shape of the lobes, consistent with the jet being precessing. As noted by Caratti o Garatti et al. (2008), the resulting pattern is too complex to be fitted with a simple precession law and suggests the presence of multiple stars perturbing the trajectory of the jet/outflow axis. The material launched in this way is bound to clear up a relatively broad solid angle along the jet/outflow axis, thus allowing the stellar photons to escape from the star neighborhoods. This can explain why in Fig. 2a the continuum emission due to scattered stellar photons is seen at large distances from the star.

An intriguing feature worthy of mention, is the "mushroomshaped" structure located a few arcsec to the NW from the jet center. This is shown in Fig. 3, where we plot the free-free continuum map by Hofner et al. (2007) and the water maser spots detected by Moscadelli et al. $(2005,2011)$ with the associated proper motions. Also shown is the nominal position of the protostar, obtained from a model fit to the $\mathrm{H}_{2} \mathrm{O}$ masers (Moscadelli et al. 2011). The latter lies along the symmetry axis (dashed line in the figure) of the "mushroom-shaped" feature, within the astrometric uncertainty of the LBT image $(\lessgtr 0, ' 1)$. The shape and orientation of this feature, compared to the other jet tracers, especially the $\mathrm{H}_{2} \mathrm{O}$ masers, strongly suggest that the $\mathrm{H}_{2}$ emission belongs to the jet and outlines a bow shock. It is worth noting the impressive similarity between the "mushroom-shaped" feature and the simulation of a magnetized jet powered by a disk wind, by Staff et al. (2010; see e.g. their Figs. 1 and 2, displaying gas density and [OI] line intensity maps). Although this simulation refers to scales of only $60 \mathrm{AU}$ from a low-mass star, one cannot rule out the possibility that more massive stars can produce analogous structures at much larger distances (in our case $\sim 4000 \mathrm{AU}$ ) and with larger sizes.

\subsection{Nature of the small-scale bipolar feature}

In the following, we focus our attention on the $K$-band bipolar structure that SWF assert to be tracing the jet very close to the circumstellar disk, seen as a dark silhouette across the two jet lobes. To check the consistency between our data and those of SWF, in Fig. 4 we present an image of the $K_{\mathrm{s}}$ band emission that covers the same region displayed in the right panel of Fig. 1 of SWF, smoothed to the same resolution (0.'3). The similarity between the two images is quite satisfactory, although the NW lobe appears slightly shifted to the south in 


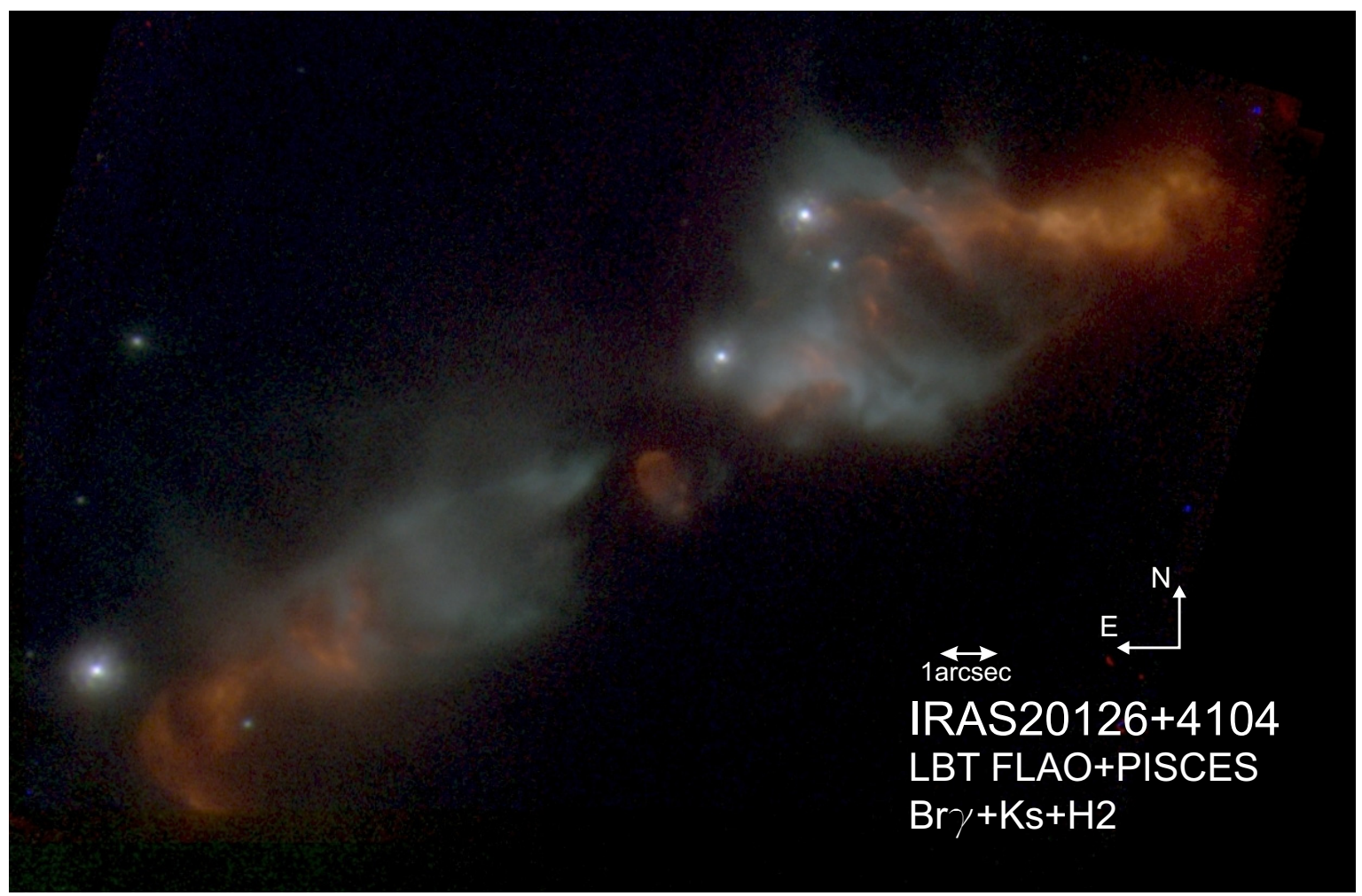

Fig. 1. Composite colour image obtained by combining the emission from the three filters used: $\mathrm{H}_{2}$ filter (red), $K_{\mathrm{s}}$ (green), and Br $\gamma$ (blue). Regions dominated by continuum emission appear white in the image, while those traced by the $\mathrm{H}_{2}$ line are red. Br $\gamma$ emission is not detected.

our data: this is not surprising, as this lobe is only barely detected in the non smoothed image (see Fig. 5a). Besides being very faint in the $2.2 \mu \mathrm{m}$ continuum, this lobe is not seen in the $\mathrm{H}_{2}$ line emission (see Fig. 5b), which might cast some doubt on its nature. However, we believe that also the NW lobe is associated with a bow-shock cavity, as well as the SE lobe, but the $\mathrm{H}_{2}$ line is not detected because of extinction. This hypothesis is supported by SWF, who estimate a larger extinction for the NW lobe (see their Table 1); moreover, the mid-IR images of De Buizer (2007) demonstrate that the NW lobe becomes progressively more prominent at longer wavelengths, where the dust opacity decreases. This result is confirmed by de Wit et al. (2009), who could image the NW lobe at $24.5 \mu \mathrm{m}$, but failed to reveal the SE lobe.

Our observations are a substantial improvement with respect to those of SWF, not only because of better angular resolution, but also because we can discriminate how much of the $K$-band emission detected by SWF is due to the $\mathrm{H}_{2}$ line. Comparison between Fig. 5a and 5b shows that the weak NW nebulosity is basically made of continuum emission, whereas the SE nebulosity is dominated by the line emission, with only a faint continuum contribution in the southwestern part of it.

With the aim to shed light on the nature of this bipolar structure, we compare our images with other disk and jet/outflow tracers from previous studies of IRAS $20126+4104$. This is done in Fig. 6, where we overlay the free-free $3.6 \mathrm{~cm}$ continuum map of Hofner et al. (2007) and the $\mathrm{H}_{2} \mathrm{O}$ and $\mathrm{CH}_{3} \mathrm{OH}$ spots detected by various authors, on our $\mathrm{H}_{2}$ image. We also plot the peaks of the $\mathrm{CH}_{3} \mathrm{CN}(12-11)$ and $\mathrm{C}^{34} \mathrm{~S}(5-4)$ emission that according to Cesaroni et al. $(1999,2005)$ trace a Keplerian circumstellar disk. These peaks have been obtained by fitting the line emission at different velocities with a 2D Gaussian (see e.g. Fig. 5 of Cesaroni et al. 1999). Since the astrometric uncertainty of the interferometric observations of $\mathrm{CH}_{3} \mathrm{CN}$ and $\mathrm{C}^{34} \mathrm{~S}$ is significantly worse $(\sim 0$.' 4$)$ than that of the VLBI and VLA observations of the masers and $3.6 \mathrm{~cm}$ continuum, we have decided to recenter the $\mathrm{CH}_{3} \mathrm{CN}$ and $\mathrm{C}^{34} \mathrm{~S}$ in the following way.

Besides the molecular line emission, an image of the $\mathrm{mm}$ continuum emission was obtained in the interferometric observations by Cesaroni et al. (1999, 2005). We made the reasonable assumption that this coincides with the position of the protostar and applied a shift to each continuum map in such a way that the continuum peak coincides with the the nominal stellar position - obtained from the model fit to the $\mathrm{H}_{2} \mathrm{O}$ masers (Moscadelli et al. 2011). The same shift was applied to the corresponding line peaks, as the $\mathrm{mm}$ line and continuum data were acquired simultaneously and the relative positional error is negligible.

Inspection of Fig. 6 confirms the scenario depicted by SWF, as the disk lies right at the border of the IR nebulosity outlined by both $\mathrm{H}_{2}$ and (weak) continuum emission. Interestingly, the $\mathrm{CH}_{3} \mathrm{OH}$ masers appear to be partly lying on the surface of the disk and partly lifting from it, parallel to the direction of the jet, as proved by their proper motions (see Fig. 3 of Moscadelli et al. 2011). Note also that the coincidence between the $\mathrm{CH}_{3} \mathrm{CN}$ and $\mathrm{C}^{34} \mathrm{~S}$ peaks with (part of) the $\mathrm{CH}_{3} \mathrm{OH}$ spots lends support to our method to improve on the astrometry of the $\mathrm{mm}$ data.

An interesting result depicted in Fig. 6 is the striking asymmetry between the SE and NW regions with respect to the protostar. While there is little doubt that the masers and free-free emission are tracing the NW lobe of the jet (apart from an $\mathrm{H}_{2} \mathrm{O}$ spot with proper motion directed towards SE, likely associated with the SE lobe), the origin of the IR emission is more doubtful. This is only barely detected to the NW, but very strong to the SE, which seems inconsistent with the orientation of the jet, 


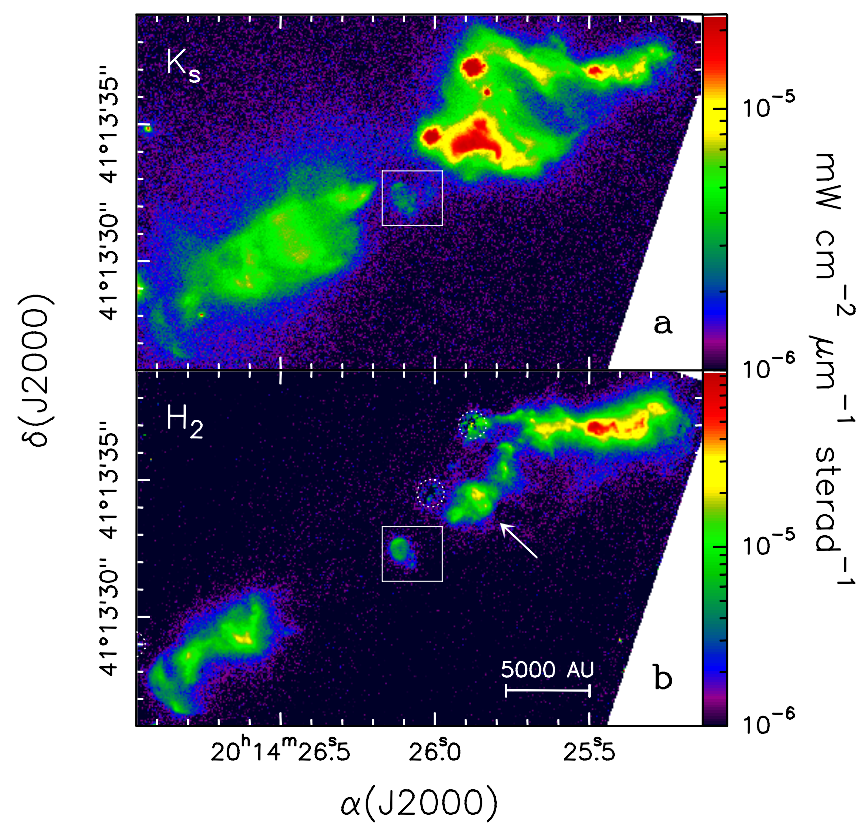

Fig. 2. a) Emission in the $K_{\mathrm{s}}$ filter of IRAS 20126+4104. The white rectangle outlines the region displayed in Fig. 5. b) Same as above, for the $\mathrm{H}_{2}$ line emission. The dotted circles indicate artifacts due to residual emission after the continuum subtraction. The arrow points at the "mushroom-shaped" structure shown in Fig. 3.

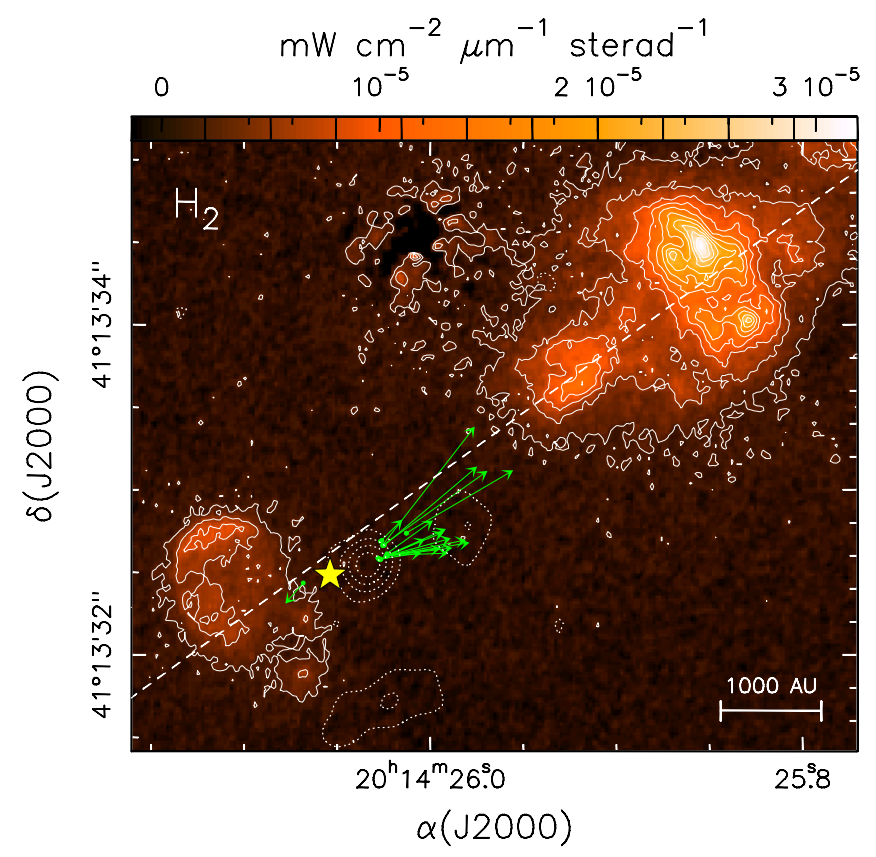

Fig. 3. $\mathrm{H}_{2}$ line emission from the IRAS $20126+4104$ region, with overlayed the $3.6 \mathrm{~cm}$ continuum map (dotted contours) from Hofner et al. (2007) and the $\mathrm{H}_{2} \mathrm{O}$ maser spots (green points) with associated proper motions (arrows) from Moscadelli et al. (2011). The solid contours correspond to the intensity levels marked in the colour scale on top of the figure. The yellow star marks the nominal position of the protostar, obtained from the model fit of Moscadelli et al. (2011) to the $\mathrm{H}_{2} \mathrm{O}$ maser positions and velocities. The dashed line is the symmetry axis of the "mushroom-shaped" $\mathrm{H}_{2}$ line emitting feature in the top right of the figure.

because the emission from the NW lobe of the jet is blue shifted and hence pointing towards the observer (Moscadelli et al. 2011;

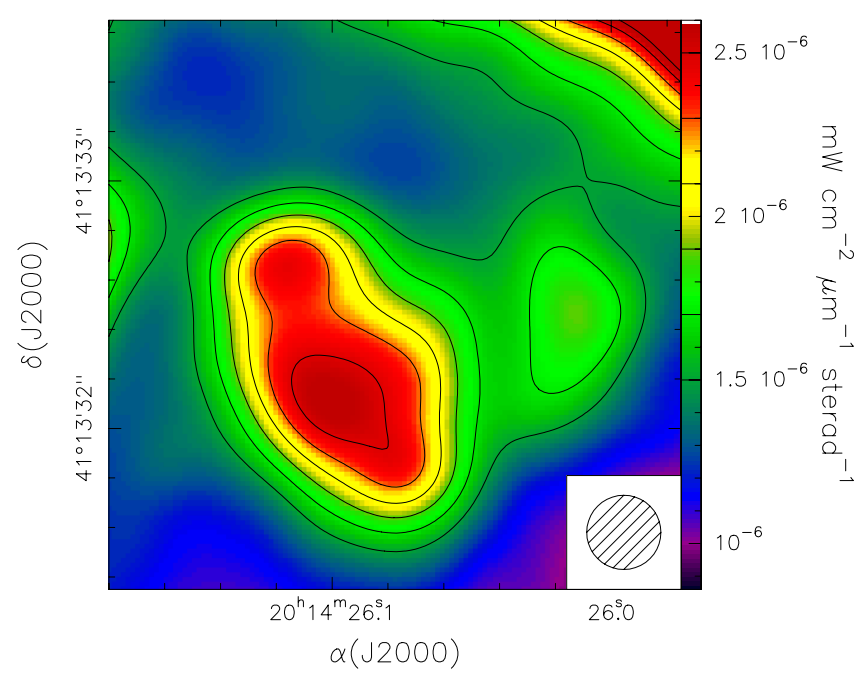

Fig. 4. Mean emission in the $K_{\mathrm{s}}$ band smoothed to the same angular resolution $\left(0{ }^{\prime} 3\right)$ as the data of SWF. The region displayed corresponds to that covered in the left panel of Fig. 1 of SWF. The angular resolution is indicated by the circle in the bottom right of the figure.

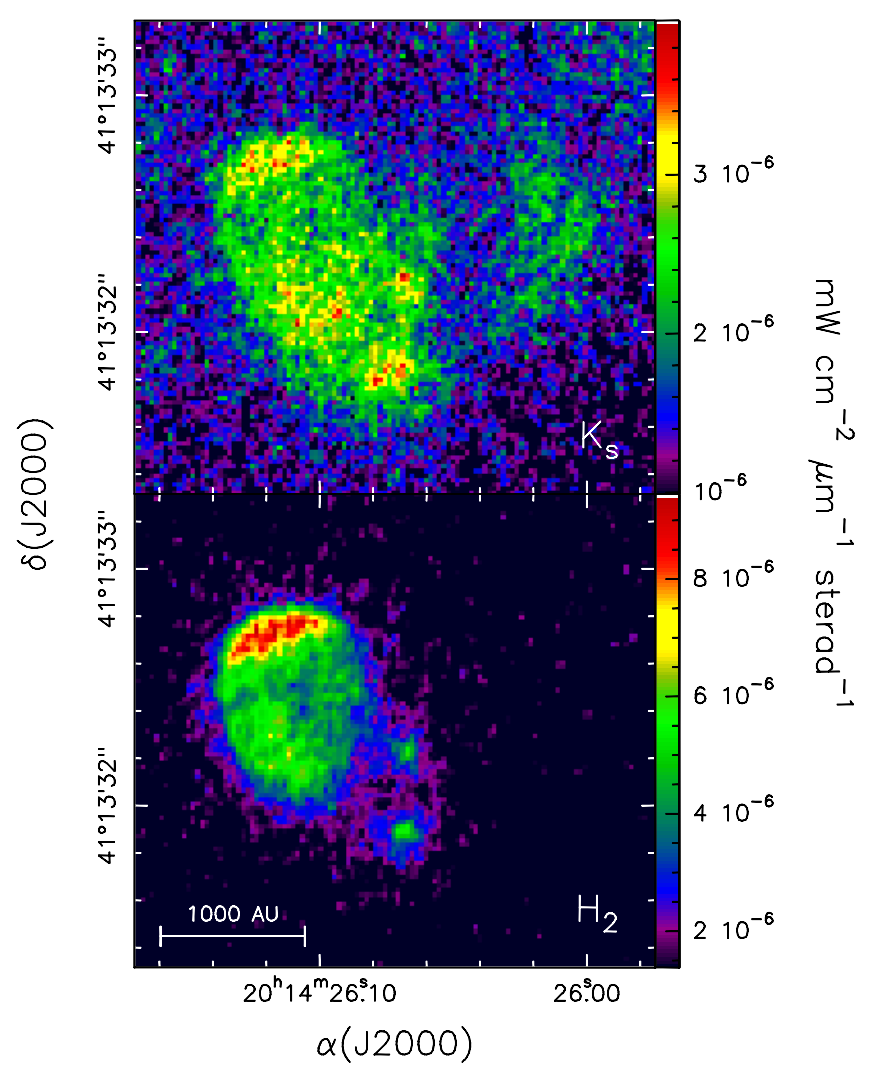

Fig. 5. Enlargment of the central region of Figs. $2 \mathrm{a}$ and $2 \mathrm{~b}$.

Cesaroni et al 2005). If the IR emission is associated with the jet, it should be more easily detected from the lobe pointing towards us (the NW lobe), than from that pointing away from us (the SE lobe), because the latter is obviously more extincted. How can one reconcile this scenario with the strong $\mathrm{H}_{2}$ line emission seen to the SE and the lack of emission to the NW?

First of all, the inclination of the jet axis with respect to the plane of the sky is very small, about $10^{\circ}$ according to Moscadelli et al. (2011). Consequently, the difference in extinction between the SE (red shifted) and NW (blue shifted) lobes cannot be justified in terms of different orientation with respect to the observer 


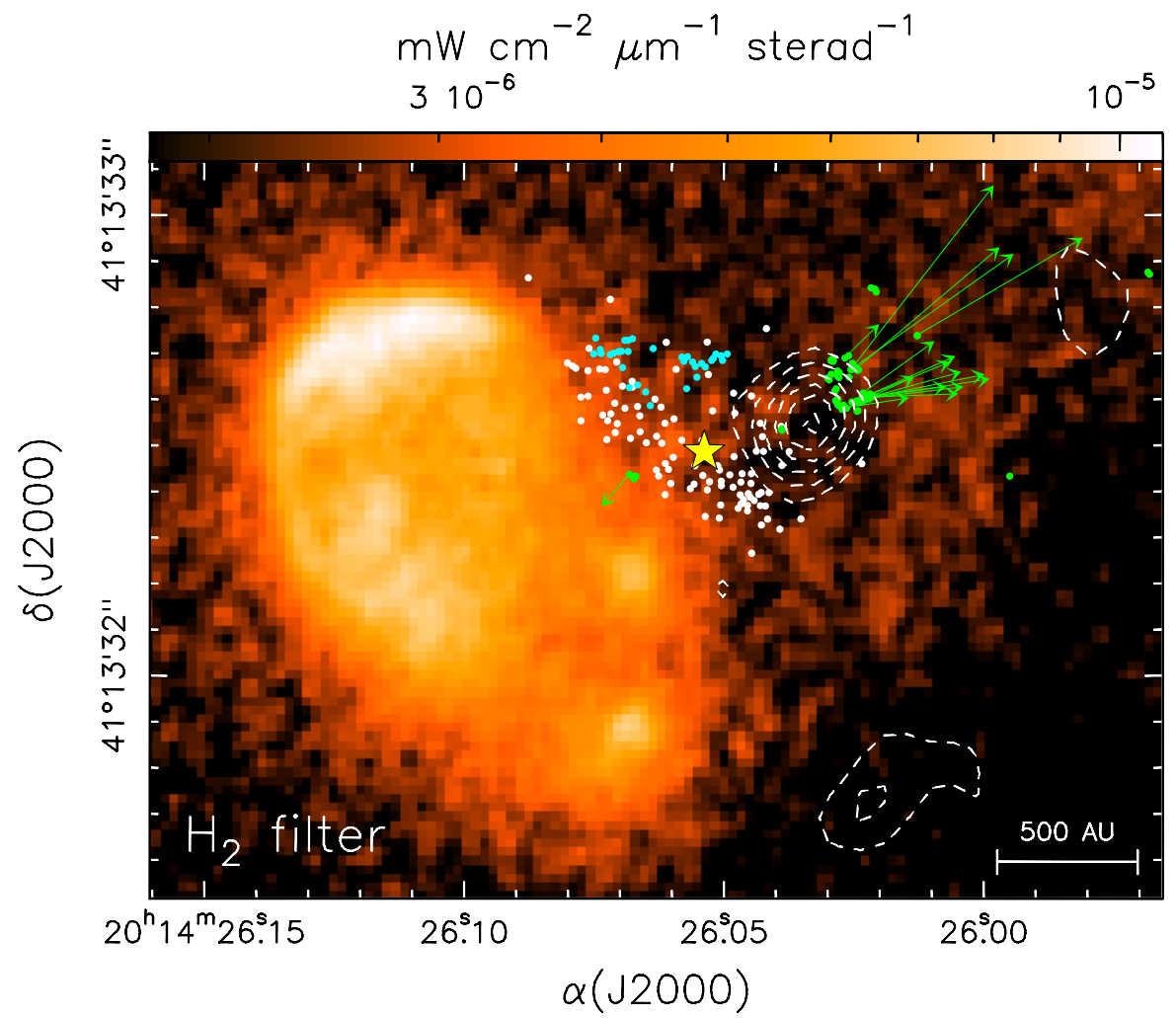

Fig. 6. Mean emission in the $\mathrm{H}_{2}$ filter (line plus continuum) of the central region of IRAS 20126+4104, with overlayed the $3.6 \mathrm{~cm}$ continuum map (dashed contours) from Hofner et al. (2007), the $\mathrm{CH}_{3} \mathrm{OH}$ maser spots (cyan points) from Moscadelli et al. (2011), and the $\mathrm{H}_{2} \mathrm{O}$ maser spots (green points) from Moscadelli et al. (2005, 2011), Edris et al. (2005), and Trinidad et al. (2005). The white points denote the peaks of the $\mathrm{CH}_{3} \mathrm{CN}(12-11)$ and $\mathrm{C}^{34} \mathrm{~S}(5-4)$ line emission from Cesaroni et al. (1999, 2005), whose position has been realigned as explained in the text. The green arrows indicate the direction and speed of the $\mathrm{H}_{2} \mathrm{O}$ maser proper motions. The yellow star marks the nominal position of the protostar, obtained from the model fit of Moscadelli et al. (2011) to the $\mathrm{H}_{2} \mathrm{O}$ masers. (the NW lobe pointing towards us, the other away from us). Therefore, the (small) jet inclination cannot explain why the $\mathrm{H}_{2}$ line (and, to some extent, continuum) emission is much brighter on one side than on the other. In our opinion, the explanation is related to the nature of ejection phenomenon. In Sect. 3.1, based on the structure of the $\mathrm{H}_{2}$ emission on a large scale, we have concluded that the jet is asymmetric, irregular, and precessing. This implies that the ejection phenomenon not only is discontinuous and nonperiodic, but also occurs at different times on the two sides with respect to the protostar. Moreover, due to precession, every new ejection has to dig its way through new, fresh circumstellar material. If the NW lobe corresponds to a more recent ejection episode than the SE lobe, it is not surprising that the jet is still pearcing a narrow passage throught the dense molecular neighborhoods. Vice versa, on the SE side an older ejection episode may have had enough time to blow the dense gas away, creating an inflated cavity from which the IR photons can escape. The absence of dense gas explains why no thermal jet and almost no maser emission is seen to the SE.

We conclude that the near-IR emission within 1 " from the protostar is coming from the bipolar jet directed SE-NW, in agreement with the interpretation of SWF. Our results demonstrate the potential of AO observations in the near-IR even in regions characterized by large local extinction and call for observations at similar wavelengths (e.g. in the $L$ and $M^{\prime}$ bands; Sridharan et al. 2007) to further the investigation of the small scale structure and stellar content of this interesting object.

Acknowledgements. It is a pleasure to thank Leonardo Testi for providing us with the TNG images used to improve the astrometric accuracy of our data. We are also grateful to the referee, Willem-Jan de Wit, for his prompt reply and constructive criticisms. We acknowledge the support from the LBT-Italian Coordination Facility for the execution of observations, data distribution and reduction.

\section{References}

Caratti o Garatti, A., Froebrich, D., Eislöffel, J., Giannini, T., \& Nisini, B. 2008, A\&A, 485, 137

Cesaroni, R., Felli, M., Testi, L., Walmsley, C. M., \& Olmi, L. 1997, A\&A, 325, 725

Cesaroni, R., Felli, M., Jenness, T., et al. 1999, A\&A, 345, 949

Cesaroni, R., Neri, R., Olmi, L., et al. 2005, A\&A, 434, 1039

Cesaroni, R., Galli, D., Lodato, G., Walmsley, C. M., \& Zhang, Q. 2007, in Protostars and Planets V, eds. B. Reipurth, D. Jewitt, \& K. Keil (Tucson: Univ. of Arizona Press), 197

De Buizer, J. M. 2007, ApJ, 654, L147

de Wit, W. J., Hoare, M. G., Fujiyoshi, T., et al. 2009, A\&A, 494, 157

de Wit, W. J., Hoare, M. G., Oudmaijer, R. D., \& Lumsden, S. L. 2010, A\&A, 515, A45

de Wit, W. J., Hoare, M. G., Oudmaijer, R. D., et al. 2011, A\&A, 526, L5

Edris, K. A., Fuller, G. A., Cohen, R. J., \& Etoka, S. 2005, A\&A, 434, 213

Esposito, S., Riccardi, A., Quirós-Pacheco, F., et al. 2010, App. Opt., 49, 174

Esposito, S., Riccardi, A., Pinna, E., et al. 2011, in Astronomical Adaptive Optics

Systems and Applications IV, eds. R. K. Tyson, \& M. Hart, Proc. SPIE, 8149, 814902

Hofner, P., Cesaroni, R., Rodríguez, L. F., \& Martí, J. 1999, A\&A, 345, 43

Hofner, P., Cesaroni, R., Olmi, L., et al. 2007, A\&A, 465, 197

Kraus, S., Hofmann, K.-H., Menten, \& Karl M. 2010, Nature, 466, 339

Krumholz, M. R., Klein, R. I., McKee, C. F., Offner, S. S. R., \& Cunningham, A. J. 2009, Science, 323, 754

Kuiper, R., Klahr, H., Beuther, H., \& Henning, Th. 2010, ApJ, 722, 1556

Kuiper, R., Klahr, H., Beuther, H., \& Henning, Th. 2011, ApJ, 732, 20

Lebrón, M., Beuther, H., Schilke, P., \& Stanke, Th. 2006, A\&A, 448, 1037

McCarthy, D. W., Jr., Ge, J., Hinz, J. L., Finn, R. A., \& de Jong, R. S. 2001, PASP, 113,353

Moscadelli, L., Cesaroni, R., \& Rioja, M. J. 2000, A\&A, 360, 663

Moscadelli, L., Cesaroni, R., \& Rioja, M. J. 2005, A\&A, 438, 889

Moscadelli, L., Cesaroni, R., Rioja, M. J., Dodson, R., \& Reid, M. J. 2011, A\&A, 526, A66

Shepherd, D. S., Yu, K. C., Bally, J., \& Testi, L. 2000, ApJ, 535, 833

Sridharan, T. K., Williams, S. J., \& Fuller, G. A. 2005, ApJ, 631, 73 (SWF)

Sridharan, T. K., Saito, M., \& Fuller, G. A., \& Kandori, R. 2007, BAAS, 39,849

Staff, J. E., Niebergal, B. P., Ouyed, R., Pudritz, R. E., \& Cai, K. 2010, ApJ, 722, 1325

Su, Y.-N., Liu, S.-Y., Chen, H.-R., Zhang, Q., \& Cesaroni, R. 2007, ApJ, 671, 571

Trinidad, M. A., Curiel, S., Migenes, V., et al. 2005, AJ, 130, 2206 